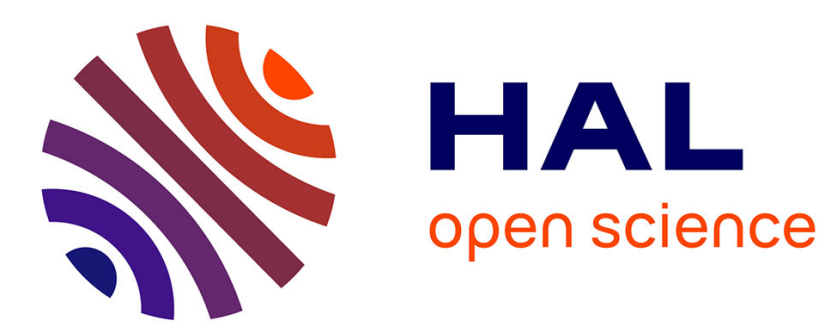

\title{
Evaluating the Gain of Directional Antennas in Linear VANETs using Stochastic Geometry
}

Paul Mühlethaler, Younes Bouchaala, Shagdar $\dagger$ Oyunchimeg, Nadjib Achir

\section{To cite this version:}

Paul Mühlethaler, Younes Bouchaala, Shagdar $\dagger$ Oyunchimeg, Nadjib Achir. Evaluating the Gain of Directional Antennas in Linear VANETs using Stochastic Geometry. PEMWN 2017 - 6th IFIP International Conference on Performance Evaluation and Modeling in Wired and Wireless Networks, Nov 2017, Paris France. pp.1-7. hal-01617937

\section{HAL Id: hal-01617937 https://hal.science/hal-01617937}

Submitted on 17 Oct 2017

HAL is a multi-disciplinary open access archive for the deposit and dissemination of scientific research documents, whether they are published or not. The documents may come from teaching and research institutions in France or abroad, or from public or private research centers.
L'archive ouverte pluridisciplinaire HAL, est destinée au dépôt et à la diffusion de documents scientifiques de niveau recherche, publiés ou non, émanant des établissements d'enseignement et de recherche français ou étrangers, des laboratoires publics ou privés. 


\title{
Evaluating the Gain of Directional Antennas in Linear VANETs using Stochastic Geometry
}

\author{
Paul Muhlethaler*, Younes Bouchaala ${ }^{\dagger}$, Oyunchimeg Shagdar ${ }^{\dagger}$, and Nadjib Achir ${ }^{\ddagger}$ \\ *INRIA EVA, Centre de Recherche de Paris, 2 Rue Simone, IFF CS 42112, 75589 Paris Cedex 12 \\ Email: paul.muhlethaler@inria.fr \\ ${ }^{\dagger}$ Institut VEDECOM, 77, rue des Chantiers, 78000 Versailles, France \\ Emails: \{younes.bouchaala, oyunchimeg.shagdar\}@vedecom.fr \\ $\ddagger$ Universite Paris 13, Sorbonne Paris Cite - L2TI, 99 Avenue J-B Clément, 93430 Villetaneuse, France \\ Email: nadjib.achir@univ-paris13.fr
}

\begin{abstract}
Maximizing the throughput of point-to-point communication has been the crux of wireless networks. In IEEE 802.11 networks, the first and prominent wireless technology, the model of point-to-point communication is still applicable today: the transmissions are between the wireless nodes and the access point, which usually serves as a gateway to the Internet. But this model is not well suited to more recent wireless systems such as Wireless Sensor Networks (WSNs) and Vehicular Ad Hoc NETworks (VANETs). In such networks, a very significant part of communication is between one node and its neighbors and simultaneous transmissions or, in other words spatial reuse, is required to insure good performance. When we consider communication from one node to its neighbor,an important metric is the density of successful simultaneous transmissions. Several studies such as [1], [2] have shown how this density of transmissions can be improved in Aloha or in CSMA networks. The aim of this paper is to show that the use of directional antennas can greatly improve the performance of the network in our neighbor-to-neighbor communication model because interference is greatly reduced. The model we build here allows a quantitative study of the performance and the improvement obtained with directional antennas to be be achieved. The study of Aloha (slotted and non-slotted) is very easy to accomplish and leads to closed formulas for the density of successful transmissions. The study of CSMA is more complex. We use a Matern selection process to mimic the behavior of CSMA in a random pattern of nodes distributed as a Poisson Point Process (PPP): each node receives a random mark and the nodes that have the smallest mark in their neighborhood are elected for transmission. Previous studies, such as [2], show that in CSMA networks, the density of successful transmissions is greatly influenced by the carrier sense detection threshold, which is one of the main parameters of CSMA. In this study we will assume that the carrier sense detection threshold is optimized to obtain the best performance of the CSMA network and our evaluations are performed under this condition. Our analytical models and our computation show that using directional antennas can lead to an improvement of up to more than $100 \%$ in the density of throughput compared to the normal use of unidirectional antennas.
\end{abstract}

Keywords-VANETs, CSMA, spatial performance, stochastic geometry.

\section{INTRODUCTION}

Until recently, the throughput of point-to-point communications was the main criterion for network performance evaluation. At the physical layer, optimizing the point-to-point throughput has always been a major objective in networks whereas at the MAC layer, minimizing the loss incurred by sharing the medium has always been a quest. When wireless networks appeared in the late 80s with the development of the IEEE 802.11 technology, this model remained partly valid; wireless nodes sent their packets to the access point and since IEEE 802.11 uses a CSMA technique, only one packet could be transmitted at a time and the paradigm of the transmission with one source and one destination was still present.

Since the birth of the Internet of Things (IoT) and the emergence of new networks such as Wireless Sensor Networks (WSNs) and Vehicular Ad hoc NETworks (VANETs), the spatial aspect of the networks has become prominent and the network can be deployed over a wide area. Simultaneous transmissions are mandatory to ensure good network performance. In VANETs, safety packets such as Car Awareness Messages (CAMs) must be sent from each vehicle to the nodes in their neighborhood. With such an application the straightforward evaluation metric is the density of successful transmissions from each node to its neighbors or, in other words, the number of successful transmissions in the network per unit of space. In an optimized network it is not sufficient that one node successfully sends its packets to its neighbors, it is also important that many other nodes in the network are able to send their packets to their neighbors within the same time interval.

Tools from stochastic geometry, such as Poisson Point Processes (PPPs), are very suitable to capture the spatial effect of new networks in the IoT such as VANETs and WSNs and to provide efficient tools to evaluate the spatial density of given events in the network (e.g successful transmissions). Moreover, they can also model random networks. As shown below, it is easy in PPPs to model the typical neighbors of a given node. Here, we adopt PPPs to model node locations and we denote the node density of our network by $\lambda$. We use the density of successful transmissions as the performance metric.

In this paper we study two different access technique : Aloha and CSMA. We also assume random fading, which simplifies the computation. Similar evaluations as those presented below are possible with other fading laws but would involve more numerical computations. Spatial Aloha networks are quite easy to model and accurately analyze in PPPs [1] mostly because the pattern of simultaneous transmissions remains a random PPP of intensity $\lambda p$ where $p$ is the transmission rate of Aloha. Thus we easily obtain closed formulas for the density of successful transmissions. The case of non-slotted Aloha 
is slightly more complex, but if we use the results of [3] we can also obtain closed formulas with a slightly different PPP. Modeling CSMA networks is much more complex. In this paper we use the Matern process to mimic the CSMA selection rule in a random PPP which can . As far as we know, there is no other technique available to directly model CSMA. Other techniques are based on simulations. We combine a classical Signal-to-Interference-and-Noise Ratio (SINR) and the selection obtained by the Matern process to analytically compute the density of successful transmissions.

The remainder of this paper is organized as follows. Section II briefly reviews related work. Section III describes the model proposed to study Aloha and CSMA. We optimize the density of successful transmissions versus the transmission probability for Aloha and versus the carrier sense threshold for CSMA. We deal with the case of omni-directional and directional antennas in order to perform the comparison. In Section IV we report the results of our analytical study and compare the results obtained with omni-directional and directional antennas. Finally, Section V concludes the paper.

\section{RELATED WORK}

In 2000, the pioneering article [4] was the first to provide an indepth study of the performance of ad hoc networks in terms of total bandwidth, taking into account the inherent spatial effect of these networks. The assumptions were a random network and a simplified model to handle interference. The traffic model was point-point with random source and destination nodes.

In the footsteps of the seminal work by Gupta and Kumar, [5] studies the gain in an ad hoc network when directional antennas are used. The work focuses on 2D networks and shows that the bandwidth gain is $\frac{2 \pi}{\alpha}$ with a directional antenna at the transmitter (with an emitting angle $\alpha$ ) and $\frac{4 \pi^{2}}{\alpha \beta}$ when we use directional antennas simultaneously in both the receiver and the transmitter with an emitting angle $\alpha$ and a receiving angle $\beta$.

In the domain of VANETs there are apparently no theoretical studies like [4], [5] and the present paper appears to be one of the first (if not the first) contribution. However, there are numerous papers which study the impact of antenna patterns in VANETs. In 1985, [6] carried out extensive measurements and showed that not only the antenna itself, but more so its position on the car and the existence of sirens and lights on the roof can significantly modify the radiation pattern of the antenna. Using ray tracing simulation, [7] studied the effects of antenna placement on car-to-car communication. The authors found that the received signal power greatly depends on the antenna position and causes huge differences in the received power (up to $30 \mathrm{~dB}$ ). Using measurements in the $5.9 \mathrm{GHz}$ frequency band, as used by the IEEE 802.11p standard, [8] investigated the effect of panoramic glass roofs on the antenna radiation pattern. The authors noted a significant negative impact due to reflections inside the glass. This causes a considerably reduced forward transmission range.

This study is also linked to performance analysis of Aloha and CSMA. The first studies which attempted to take spatial reuse into account for Aloha appeared in the 1980s and a model for slotted Aloha was later introduced in 1988 by Ghez, Verdu and Schwartz [9]. This study was refined in [1] where the performance of a network based on this same model was more accurately evaluated. In particular,fooof the capture probability and the density of successful transmissions were computed when the distance between the source and the destination was known. These evaluations were possible due to the complete and stateless randomization of the transmitting nodes in Aloha networks. As for CSMA, the interference issue was raised in [10] for a linear network of randomly positioned vehicles, however, the study only considered the nearest interferer case. The pattern of simultaneous transmissions in CSMA was first evaluated in [11] using the Matern selection process [12]. Another similar process was used in [13] in order to evaluate interferences in CSMA, however, the study did not assess the overall network throughput. The model initially developed in [11] and subsequently enhanced in [14], is extended in this current work. More recent studies show the prime importance of adapting the carrier sense threshold in CSMA networks [2] and [15] proposes an algorithm to do so.

\section{SYSTEM MODEL}

In this paper we consider a homogeneous Poisson-PointProcess (PPP) $\Phi$ extended over a 1D infinite line $\mathcal{S}=\mathbb{R}$. As Vehicular Ad-hoc NETworks (VANETs) are generally linear networks, they are usually modeled by $1 \mathrm{D}$ networks. As previously stated, we denote the intensity of the PPP by $\lambda$.

We assume that the power of a transmission over a distance $r$ is affected by a power-law decay $1 / r^{\beta}$ where $\beta$ varies between 2 and 6 depending on the propagation conditions and a random fading $F$. The power received at distance $r$ from the source node is thus $P=\frac{P_{0} F}{l(r)}$ and we set $P_{0}=1$ with $l(r)=r^{\beta}$. We also adopt a Rayleigh fading i.e., exponentially distributed with parameter $\mu$ and thus a mean of $1 / \mu$.

We use the well-accepted SIR (Signal-to-Interference-Ratio) model with a capture threshold $T$. We omit thermal noise but it could be easily added, as explained below. An even more realistic model than the SIR based on a graded SIR model using Shannon's law is possible in our framework though with an increased computational cost. In other words, a successful transmission occurs when the ratio of the received signal divided by the interference (i.e., the other concurrent transmissions) will be greater than $T$.

\section{A. Model for directional transmission}

As we are dealing with vehicles moving along roads, our vehicular network consists in 1D lines which can be modeled by $\mathbb{R}$. We assume that the vehicles send their packets such as CAMs (Car Awareness Messages) or DENMs (Decentralized Emergency Notification Messages) only in one direction. For instance, on motorways we can assume that the messages are only sent downstream. If we assume that the vehicles follow a one-dimensional Poisson Point Process of rate $\lambda$, the consequence of using a directional antenna is that another vehicle will interfere with the current transmission with probability 0.5 and with the same probability the vehicle will not interfere. This is true if we assume here that half of the vehicles are moving in one direction while the other half are moving in the opposite direction. Thus with omni-directional antenna the population of interfering vehicles must be selected by the access scheme in a PPP of rate $\lambda$ whereas with directional antennas the population of interfering vehicles must be selected in a PPP of rate $\lambda / 2$. In these two populations the Aloha protocol uses a random selection to select the transmitters 
whereas in CSMA the selection relies on the carrier sense threshold and the backoff timers. This will be explained in the following sections.

\section{B. Slotted and non-slotted Aloha}

In Aloha, the MAC scheme consists in a random selection of the transmitting nodes. Each node will transmit its packets with a given transmission probability $p$. The protocol can be slotted if we have given time-slots or non-slotted when we use asynchronous transmissions. We use the results of [1] for slotted Aloha and [3] for non-slotted Aloha.

Proposition III.1. For slotted Aloha, the density of successful transmissions for omni-directional antennas is:

$$
\lambda p e^{-\frac{2 \pi r \lambda p T^{\frac{1}{\beta}}}{\beta \sin \left(\frac{\pi}{\beta}\right)}}
$$

and the maximum value of this density is:

$$
\frac{\beta \sin (\pi / \beta) e^{-1}}{2 \pi r T^{\frac{1}{\beta}}}
$$

For directional antennas, the density of successful transmissions is:

$$
\lambda p e^{-\frac{\pi r \lambda p T^{\frac{1}{\beta}}}{\beta \sin \left(\frac{\pi}{\beta}\right)}}
$$

and the maximum value of this density is:

$$
\frac{2 \beta \sin (\pi / \beta) e^{-1}}{\pi r T^{\frac{1}{\beta}}}
$$

The proof of Proposition III.1 can be found in [1] with the only change being that the computation of the shot-noise is done in $\mathbb{R}$ instead of $\mathbb{R}^{2}$.

Proposition III.2. Here to obtain closed formulas, we use a slightly different model, the Poisson Rain model, see [3]. This means that at each transmission the positions of the vehicles are re-sampled. This model provides very good results even if we assume that the vehicles' positions are not re-affected after each transmission. The advantage of the Poisson Rain model is that it gives closed and compact formulas. For nonslotted Aloha, the density of successful transmissions for omnidirectional antennas is:

$$
\lambda p e^{-\frac{4 \pi r \lambda p T^{\frac{1}{\beta}}}{(\beta+1) \sin \left(\frac{\pi}{\beta}\right)}}
$$

and the maximum value of this density is:

$$
\frac{(\beta+1) \sin (\pi / \beta) e^{-1}}{4 \pi r T^{\frac{1}{\beta}}}
$$

For directional antennas, the density of successful transmissions is:

$$
\lambda p e^{-\frac{2 \pi r \lambda p T^{\frac{1}{\beta}}}{(\beta+1) \sin \left(\frac{\pi}{\beta}\right)}}
$$

and the maximum value of this density is:

$$
\frac{(\beta+1) \sin (\pi / \beta) e^{-1}}{2 \pi r T^{\frac{1}{\beta}}}
$$

The proof of Proposition III.1 can be found in [3] again with the only change being that the shot-noise is computed in $\mathbb{R}$ instead of $\mathbb{R}^{2}$. Moreover to obtain this simple formula for nonslotted Aloha, we have to assume a model of Poisson rain nodes but this model also seems to be accurate in traditional Poisson Point Processes, see [3].

\section{CSMA}

To mimic the CSMA selection process we use a Matern selection process. In this scheme each node $X_{i} \in \Phi$ receives a random mark $m_{i}$ and the process selects the node with the lowest mark in its neighborhood. This neighborhood is defined as follows. We denote by $F_{i, j}$ the fading for a transmission between $X_{i}$ and $X_{j}$ and we also introduce the carrier sense threshold $P_{c s}$ of our CSMA protocol. In our definition the neighborhood of $X_{i}$ is $\mathcal{V}\left(X_{i}\right)=\left\{X_{j} \in X_{i} F_{i, j} / l\left(\mid X_{i}-\right.\right.$ $\left.\left.X_{j} \mid\right)>P_{c s}\right\}$. A node, say $X_{i}$ will be selected by the Matern selection process if and only if $\forall X_{j} \in \mathcal{V}\left(X_{i}\right) m_{i}<m_{j}$, i.e $X_{i}$ has the lowest mark $m_{i}$ in its neighborhood. It is easy to verify that a selection process is well defined by this property. In CSMA networks, the selection process is actually performed according to the back-off value; this back-off is decremented by the node during idle periods until the transmission of the packet. Thus the node with the lowest back-off time in its neighborhood will be chosen to transmit. The random marks of the Matern selection process can be interpreted as the backoff times of the waiting nodes. However, in a real CSMA network when a node transmits its packet, the other nodes in its neighborhood have already been eliminated and will no longer be able to eliminate other nodes. This is not the case in the Matern selection process, which produces an over-elimination and thus underestimates the density of transmissions. This is illustrated in Figure III.1. Following the Matern selection process, node $i$ has correctly eliminated node $k$; even so, node $k$ is still able to eliminate node $l$. In contrast, in a real CSMA system, once node $i$ has eliminated node $k$, node $k$ will no longer be able to eliminate any other neighbor. We note that in Figure III.1 we have used directional antennas.
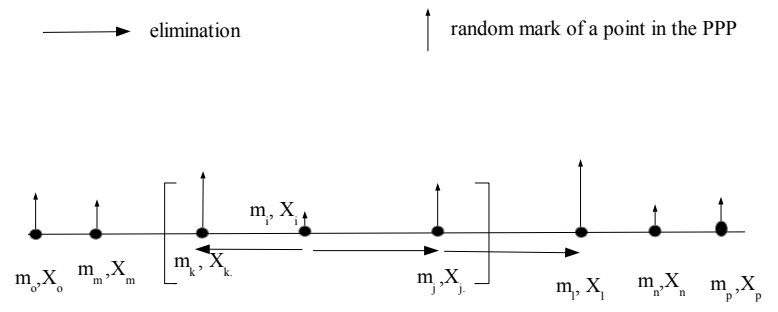

Fig. III.1. Matern CSMA selection process and an example of overelimination.

We note the medium access indicator of node $X_{i} e_{i}=$ $\mathbb{I}\left(\forall X_{j} \in \mathcal{V}\left(X_{i}\right) m_{i}<m_{j}\right)$

Proposition III.3. The mean number of neighbors of a node is:

$$
\mathcal{N}=\lambda \int_{\mathcal{S}} P\left\{F \geqslant P_{C s} l(|x|)\right\} d x .
$$


With omni-directional antennas we have:

$$
\mathcal{N}_{o}=\frac{2 \lambda \Gamma(1 / \beta)}{\beta\left(P_{c s} \mu\right)^{1 / \beta}} .
$$

With directional antennas we have:

$$
\mathcal{N}_{d}=\frac{\lambda \Gamma(1 / \beta)}{\beta\left(P_{c s} \mu\right)^{1 / \beta}} .
$$

This result is straightforward. Let $F_{j}^{0}$ be the fading between the transmitting node at the origin $X_{i}$ and the receiving node $X_{j}$. This is just the application of Slivnyak's theorem and Campbell's formula, see [16], [14]

$$
\begin{array}{rlrl}
\mathcal{N}_{\{o, d\}} & = & E^{0}\left[\sum_{X_{j} \in \phi} \mathbb{I}\left(F_{j}^{0} l\left(\left|X_{j}-X_{i}\right|\right) \geqslant P_{c s}\right]\right. \\
= & \quad \lambda_{\{o, d\}} \int_{\mathcal{S}} P\left\{F \geqslant P_{c s} l(|x|)\right\} d x
\end{array}
$$

with $\lambda_{o}=\lambda$ for omni-directional antennas and $\lambda_{d}=\lambda / 2$ for directional antennas. An immediate computation yields the explicit value of $\mathcal{N}_{\{o, d\}}$.

Proposition III.4. The probability $p$ that a given node $X_{0}$ transmits i.e., $e_{0}=1$ is:

$$
p=\mathbf{E}^{0}\left[e_{0}\right]=\frac{1-e^{-\mathcal{N}}}{\mathcal{N}} .
$$

Proof: We compute the probability of a given node at the origin with the mark $m=t$ being allowed to transmit. Deconditioning on $t$ provides the result, see [14] for details. $\mathcal{N}$ is either $\mathcal{N}_{\{o, d\}}$ depending on whether we use directional antenna.

If $p$ is close to 1 , then the carrier sense imposes no restriction on transmission. On the other hand, if $p$ is close to 0 , then the carrier imposes a severe restriction on transmission.

Proposition III.5. The probability that $X_{0}$ transmits given that there is another node $X_{j} \in \Phi$ at distance $r$ is $p_{r}$ with

$$
p_{r}=p-e^{-P_{c s} \mu l(r)}\left(\frac{1-e^{-\mathcal{N}}}{\mathcal{N}^{2}}-\frac{e^{-\mathcal{N}}}{\mathcal{N}}\right)
$$

Proof: The proof is the same as that of Proposition III.4.

Proposition III.6. Let us suppose that $X_{1}$ and $X_{2}$ are two points in $\Phi$ such that $\left|X_{1}-X_{2}\right|=r$. We suppose that node $X_{2}$ is retained by the selection process. The probability of $X_{1}$ also being retained is:

$$
\begin{gathered}
h_{\{o, d\}}(r)= \\
\frac{2}{\frac{2}{b_{\{o, d\}}(r)-\mathcal{N}_{\{o, d\}}}\left(\frac{1-e^{-\mathcal{N}_{\{o, d\}}}}{\mathcal{N}_{\{o, d\}}}-\frac{1-e^{-b_{\{o, d\}}(r)}}{b_{\{o, d\}}(r)}\right)\left(1-e^{-P_{c s} \mu l(r)}\right)} \\
\frac{1-e^{-\mathcal{N}}\{o, d\}}{\mathcal{N}}-e^{-P_{c s} \mu l(r)}\left(\frac{1-e^{-\mathcal{N}}\{o, d\}}{\mathcal{N}_{\{o, d\}}^{2}}-\frac{e^{-\mathcal{N}}\{o, d\}}{\mathcal{N}_{\{o, d\}}}\right)
\end{gathered}
$$

with for omni-directional antennas

$$
b_{o}(r)=2 \mathcal{N}_{o}-\lambda \int_{-\infty}^{\infty} e^{-P_{c s} \mu(l(|x|)+l(|r-x|)} d x .
$$

and for directional antennas

$$
b_{d}(r)=2 \mathcal{N}_{d}-\frac{\lambda}{2} \int_{-\infty}^{\infty} e^{-P_{c s} \mu(l(\tau)+l(|r-\tau|))} d \tau
$$

Proof: The proof can be found in [14]. The formula is a simple adaptation of the $2 \mathrm{D}$ case to the $1 \mathrm{D}$ case and is left to the reader. Careful attention must be paid to distinguish the directional antenna and omni-directional antenna cases.

Proposition III.7. Given the transmission of a packet, we denote by $p_{c}\left(r, P_{c s}\right)$ the probability this packet being successfully received at distance $r$ in a CSMA system (modeled by a Matern selection process with a carrier sense threshold $P_{c s}$ ) and with a capture threshold $T$. We have:

$$
p_{c}^{\{o, d\}}\left(r, P_{c s}\right) \simeq \exp \left(-\lambda \int_{-\infty}^{\infty} \frac{h_{\{o, d\}}(\tau)}{1+\frac{l(|r-\tau|)}{T l(r)}} d \tau\right)
$$

Proof: Assuming a packet is transmitted, $p_{c}\left(r, P_{c s}\right)$ denotes the probability of this packet being successfully received at distance $r$ in a CSMA system using a Matern selection process with a carrier sense threshold $P_{c s}$ and with a capture threshold $T$.

The idea is to consider a transmitter at the origin and to evaluate the probability of successful reception by a receiver located at distance $r$. We condition the reception of a packet by the presence of another transmitting node at distance $\tau$. According to proposition III.6, the density of such nodes is $\lambda h(\tau)$. We obtain the result by integrating on $\tau$. The details of the proof can be found in [14] for 2D networks. The 1D network case is a simple adaptation of the $2 \mathrm{D}$ case.

It is easy to add thermal noise $W$ to the model. The expression of $p_{c}\left(r, P_{c s}\right)$ must then be multiplied by $\mathcal{L}_{W}(\mu T l(r))$ where $\mathcal{L}_{W}($.$) is the Laplace Transform of the noise.$

Proposition III.8. The spatial density of successful transmissions is thus:

$$
\lambda p p_{c}\left(r, P_{c s}\right)
$$

$p_{c}\left(r, P_{c s}\right)$ is $p_{c}^{o}\left(r, P_{c s}\right)$ for omni-directional antennas and $p_{c}^{d}\left(r, P_{c s}\right)$ for directional antennas.

Proof: Proposition III.8 is just the exploitation of Propositions III.4 and III.7.

\section{RESULTS OF THE MODEM AND COMPARISON DIRECTIONAL/OMNI-DIRECTIONAL ANTENNAS}

We study the transmissions for pairs of source-neighbor nodes We know that the average distance between one node and its neighbor node is $r=1 / \lambda$, thus we adopt the value of $r$ in the following.

The aim of our adaptive algorithm will be to ensure that $P_{c s}$ is tuned so that the spatial density of successful transmissions as defined in Proposition III.8 is optimized with respect to the spatial density of nodes $\lambda$ in the Poisson Point Process.

\section{A. Results with Aloha}

In Figure IV.1 we present the density of successful transmission for slotted Aloha with omni directional and directional antennas for different of $p$. We use the following parameters 
$\lambda=0.1, r=10$ and $\beta=2$. The gain when we use a directional antenna (rather than an omni-directional antenna) is 2.7 for $p=0.2,7.5$ for $p=0.4$ and up 30 to when $p=0.6$.

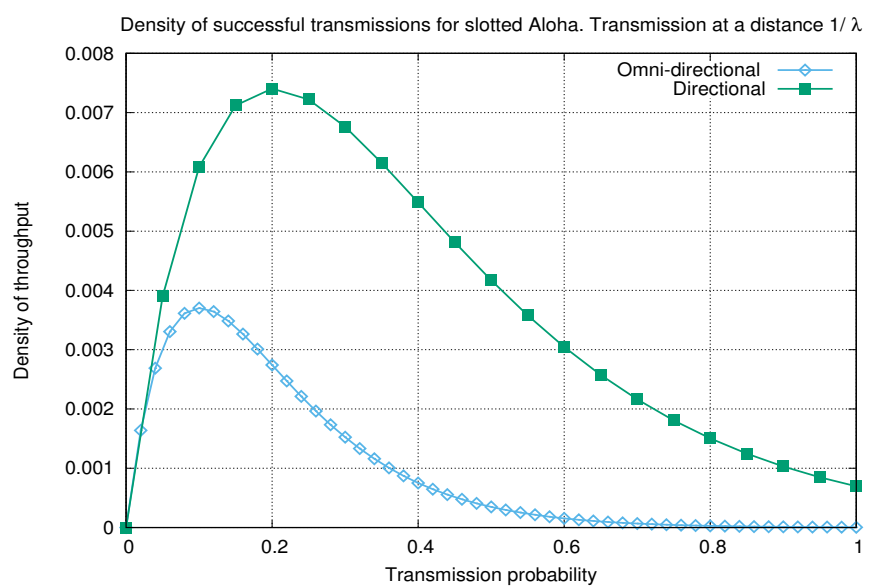

Fig. IV.1. Density of successful transmissions versus $p$ with $T=10, \beta=2$ (Slotted Aloha).

In Figure IV.2, we present the density of successful transmissions for non-slotted Aloha with omni directional and directional antennas for different values of $p$ and $\lambda=0.1$, $r=10$ and $\beta=2$. We note again a significant gain if directional antennas are used. A comparison of Figure IV.1 and Figure IV.2 allows one to compare the performance of nonslotted and slotted Aloha. If $p$ is optimized for each scheme,the gain of slotted Aloha over non-slotted Aloha is around 1.35 for directional antennas and omni-directional antennas.

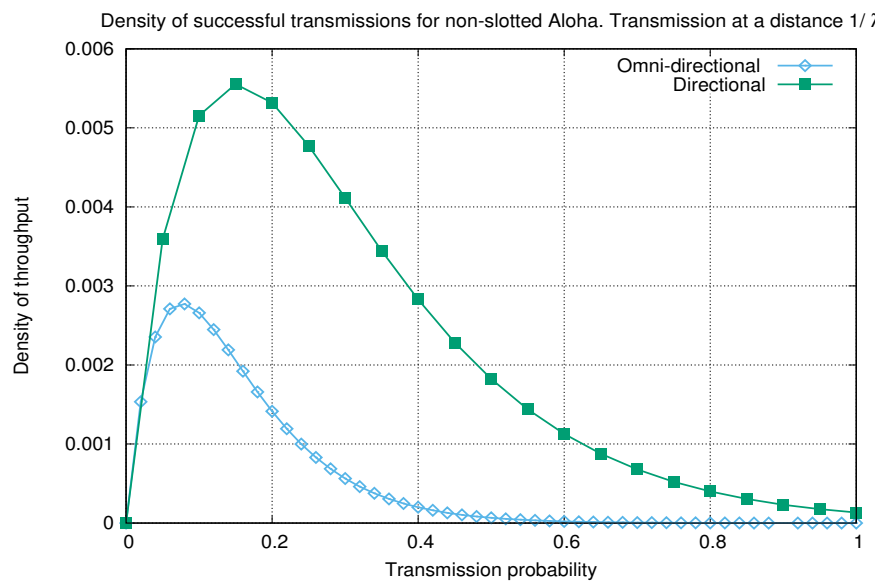

Fig. IV.2. Density of successful transmissions versus $p$ with $T=10, \beta=2$ (Non-slotted Aloha).

In order to make the best use of our MAC protocols, we optimize Aloha in $p$ with directional and omni-directional antennas and we present the value of the density of successful transmissions with this optimization. The results of these computations are given in Figure IV.3, where we vary the transmission decay $\beta$. The gain of the directional antenna over the omni-directional antenna is uniformly equal to 2 .

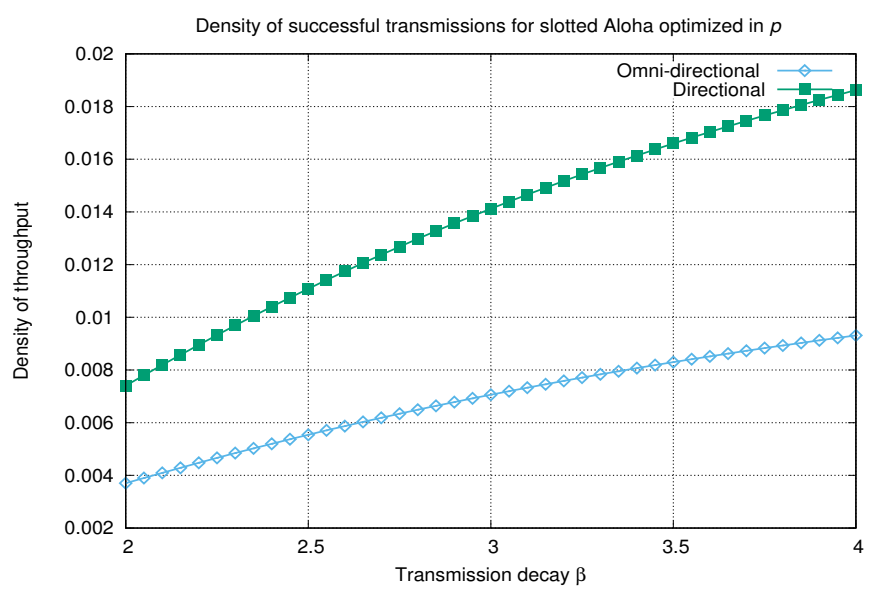

Fig. IV.3. Density of successful transmissions optimized in $p$ with $T=10$, $\beta=2$.

Figure IV.4 gives the density of successful transmissions for non-slotted Aloha when we vary the transmission decay $\beta$. The gain of the directional antenna over the omni-directional antenna is again uniformly equal to 2 .

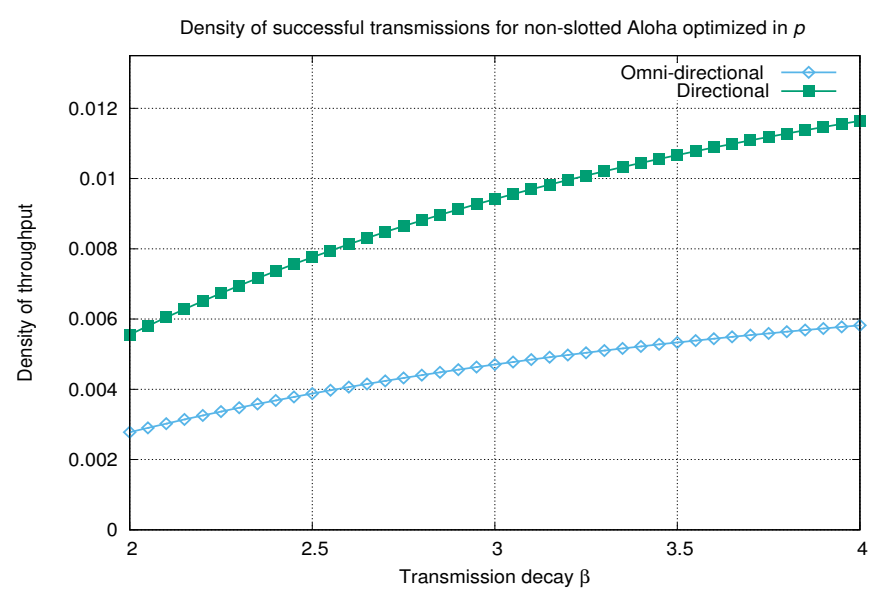

Fig. IV.4. Density of successful transmissions versus $p$ with $T=10, \beta=2$ (Non-slotted Aloha).

\section{B. Results with CSMA}

We still use the following parameters $\lambda=0.1, r=10$ and $\beta=2$ if not otherwise specified. In Figure IV.5 we study the density of successful transmission for CSMA when we use omni-directional and directional antennas. Using directional antennas provides better performance than using omni-directional antennas except when the carrier detection threshold is high. For $P_{c s}=0.001$, the gain obtained when we use directional rather than of omni-directional antennas is 1.44 and 1.15 for $P_{c s}=0.002$. For $P_{c s}=0.0032$, directional and omni-directional antennas have a similar density of successful transmissions. For $P_{c s}>0.0032$, omni-directional antennas give better performances than directional antennas. 


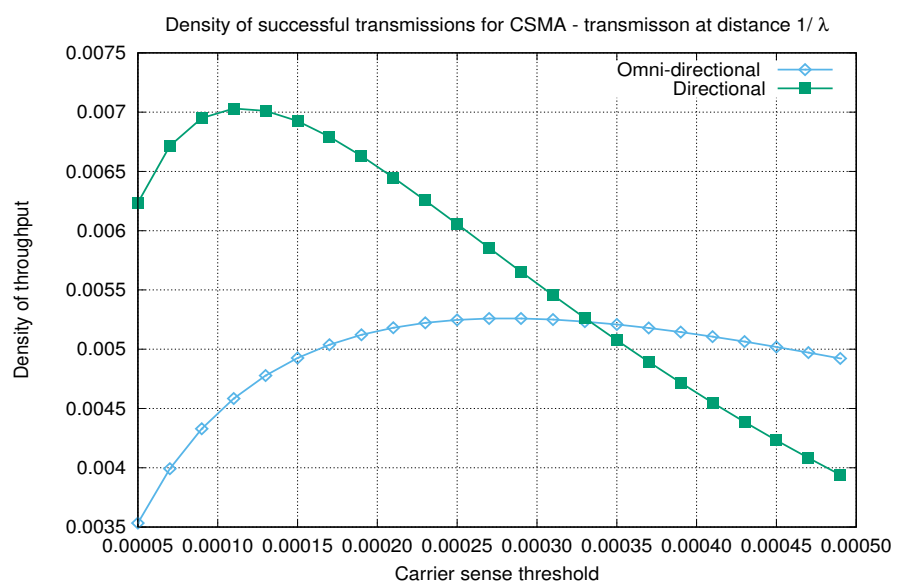

Fig. IV.5. Density of successful transmissions for different values of $P_{c s}$ for CSMA with omni-directional and directional antennas $(\lambda=0.1, r=10$, $T=10$ and $\beta=2$ ).

To obtain the best performance we study the optimization of CSMA (with directional and omni-directional antennas) with respect to the carrier sense detection threshold $P_{c s}$. The result of this study is presented in Figure IV.6. The density of successful transmissions is increased by a factor of 1.94 when $\beta=1.5$ and by a factor of 1.47 when $\beta=3$.

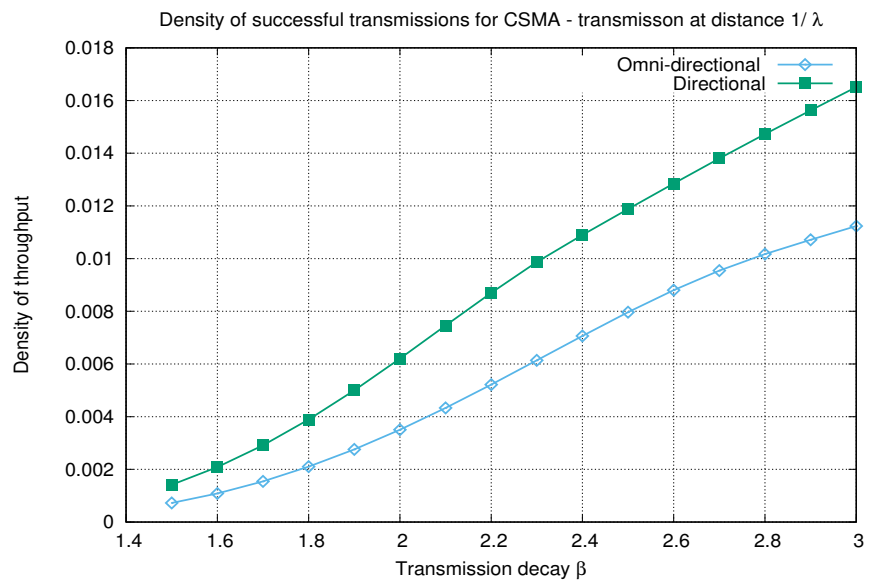

Fig. IV.6. Density of successful transmissions when the carrier sense detection threshold is optimized versus $\beta(\lambda=0.1, r=10, T=10)$.

In Figure IV.7, we study the density of successful transmission (when CSMA is optimized with respect to $P_{c s}$ ) with respect to the capture threshold $T$. We observe that the gain when we use directional antennas rather than of omni-directional antennas is 1.97 when $T=1$ and only 1.68 when $T=10$.

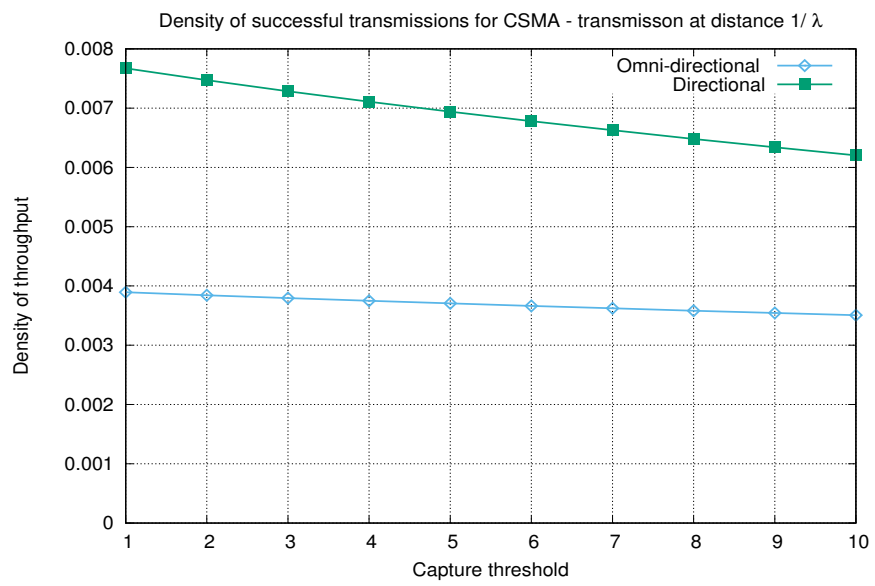

Fig. IV.7. Density of successful transmissions when the carrier sense detection threshold is optimized versus $T$. $(\lambda=0.1, r=10, T=10$ and $\beta=2)$.

\section{CONClusion}

In this paper, we present a simple stochastic model based on the Signal-to-Interference Ratio (SIR) to evaluate the gain when we use directional antennas instead of omni-directional antennas in a VANET. We assume that the vehicles in our VANET are located according to a homogeneous Poisson Point Process of density $\lambda$. The decay of the transmission signal follows a power law and we assume that there is also Rayleigh fading. We compute the density of successful transmissions from a given node to a neighbor node at distance $r$. In our numerical results $r$ is chosen to be the average distance from one node to its closest neighbor.

We adopt Aloha (slotted and non-slotted) and CSMA as access schemes. For Aloha we have obtained simple closed formulas. We have also obtained explicit formulas for CSMA but which require more intensive computation. For any given transmission probability $p$, Aloha with directional antennas has a significantly larger density of successful transmissions than Aloha with omni-directional antennas. When optimized in $p$, the gain in density of successful transmission of Aloha when we use directional instead of omni-directional antennas is exactly 2. In CSMA, a gain most often occurs when we use directional rather than omni-directional antennas. This also true when the carrier sense detection threshold is optimized. The gain obtained when when use directional antennas belongs to the interval $[1.47,1.97]$ depending on the values of the parameters of the model $\beta$ and $T$.

\section{REFERENCES}

[1] F. Baccelli, B. Blaszczyszyn, and P. Muhlethaler, "An aloha protocol for multihop mobile wireless networks," Information Theory, IEEE Transactions on, vol. 52, no. 2, pp. 421-436, Feb 2006.

[2] P. M. Nadjib Achir, Younes Bouchaala and O. Shagdar, "Optimisation of spatial csma using a simple stochastic geometry model for 1d and 2d networks," in IWCMC 2016, September 5-9th, 2016, Paphos, Cyprus 2016.

[3] B. Blaszczyszyn and P. Muhlethaler, "Stochastic analysis of nonslotted aloha in wireless ad-hoc networks," in 2010 Proceedings IEEE INFOCOM, March 2010, pp. 1-9.

[4] P. Gupta and P. R. Kumar, "The capacity of wireless networks," IEEE Transactions on Information Theory, vol. 46, no. 2, pp. 388-404, Mar 2000 . 
[5] S. Yi, Y. Pei, and S. Kalyanaraman, "On the capacity improvement of ad hoc wireless networks using directional antennas," in Proceedings of the 4th ACM International Symposium on Mobile Ad Hoc Networking \&Amp; Computing, ser. MobiHoc '03. New York, NY, USA: ACM, 2003, pp. 108-116. [Online]. Available: http://doi.acm.org/10.1145/778415.778429

[6] R. L. Jesch, "Measured vehicular antenna performance," IEEE Transactions on Vehicular Technology, vol. 34, no. 2, pp. 97-107, May 1985.

[7] L. Reichardt, T. Fugen, and T. Zwick, "Influence of antennas placement on car to car communications channel," in 2009 3rd European Conference on Antennas and Propagation, March 2009, pp. 630-634.

[8] A. Kwoczek, Z. Raida, J. Lk, M. Pokorny, J. Puskel, and P. Vgner, "Influence of car panorama glass roofs on car2car communication (poster)," in 2011 IEEE Vehicular Networking Conference (VNC), Nov 2011, pp. 246-251.

[9] S. Ghez, S. Verdu, and S. Schartz, "Stability properties of slotted Aloha with multipacket reception capability," IEEE Trans. Automat. Contr., vol 7, pp. 640-648, 1988.

[10] P. Jacquet and P. Muhlethaler, "Mean number of transmissions with csma in a linear network," in 2010 IEEE 72nd Vehicular Technology Conference: VTC2010-Fall, 69 September 2010, Ottawa, Canada 2010.

[11] P. Muhlethaler and A. Najid, "Throughput optimization in multihop csma mobile ad hoc networks," in EW 2004. The 5th European Wireless Conference, February 24 - 27. Barcelona 2004.

[12] D. Stoyan, W. S. Kendall, and J. Mecke, Stochastic geometry and its applications. 2nd edition. Wiley, 1995.

[13] A. Busson and G. Chelius, "Point processes for interference modeling in csma/ca ad-hoc networks," in Conference: Proceedings of the 6th ACM International Workshop on Performance Evaluation of Wireless Ad Hoc, Sensor, and Ubiquitous Networks, PE-WASUN 2009, October 28-29 2009, Tenerife, Canary Islands, Spain, 2009.

[14] F. Baccelli and B. Błaszczyszyn, Stochastic Geometry and Wireless Networks, Volume II - Applications, ser. Foundations and Trends in Networking. NoW Publishers, 2009, vol. 4, No 1-2.

[15] P. Muhlethaler, Y. Bouchaala, O. Shagdar, and N. Achir, "A simple stochastic geometry model to test a simple adaptive csma protocol: Application for vanets," in 2016 International Conference on Performance Evaluation and Modeling in Wired and Wireless Networks (PEMWN), Nov 2016, pp. 1-6.

[16] F. Baccelli and B. Błaszczyszyn, Stochastic Geometry and Wireless Networks, Volume I - Theory, ser. Foundations and Trends in Networking. NoW Publishers, 2009, vol. 3, No 3-4. 